\title{
Laws are not descriptions
}

\author{
Federico Laudisa \\ Department of Human Sciences, University of Milan Bicocca \\ Piazza dell'Ateneo Nuovo 1, 20126 Milan, Italy
}

\begin{abstract}
The view that takes laws of nature to be essentially nothing more than descriptions of facts is still rather popular. The present paper, on the contrary, defends the claim that the only real motivation for defending a descriptive view of laws - the quest for ontological parsimony - entails too high a price to pay in philosophical terms. It is argued that nomic primitivism, namely the alternative option that takes laws to be primitive fundamental entities in our ontology, is decisively more appealing, since it is the crucial role assigned to laws what makes a scientific theory of natural phenomena a system rather than a list. Finally, the implications that nomic primitivism might have on the issue of the status of the wave function in that particular formulation of quantum mechanics known as Bohmian mechanics are considered.
\end{abstract}




\section{Introduction}

\begin{abstract}
About ten years ago I spoke with Einstein about the astonishing fact that so many ministers of various denominations are strongly interested in the theory of relativity. Einstein said that according to his estimation there are more clergymen interested in relativity than physicists. A little puzzled I asked him how he would explain this strange fact. He answered, a little smiling, "Because clergymen are interested in the general lawe of nature and physicists, very often, are not."
\end{abstract}

Frank 1949, p. 349

According to a common sense intuition, the very idea of natural law entails that the law governs the phenomena falling under its domain of application. According to this intuition, the true role of a natural law is exactly that of providing for the relevant phenomena such 'governing' things as order, structure, evolution over time, and the like. Bas van Fraassen, just to mention one out of many possible examples, echoes such a widely felt intuition when in his Laws and Symmetry he recalls that one of the main tasks traditionally attributed to natural science is exactly "to state the laws which the things in the universe obey" (van Fraassen 1989 , p. 18, emphasis added $)^{1}$. Although it does not prove anything per se and needs to be supplemented by robust arguments in order to be convincing, this intuition seems to have an undeniable appeal and van Fraassen's wording is especially apt in our case: when there is someone or something that governs, there is always also someone or something that obeys and, in the case of natural phenomena, for them to obey means that they are constrained by some form of necessity or 'nomicity' (be it deterministic or indeterministic): we tend to think that a freely falling stone cannot choose whether its motion will be uniform or accelerated!

As we know from the history of philosophy, however, it is highly controversial whether we can legitimately project such necessity or nomicity onto the world or we should limit ourselves to locate them within the knowing subject. For instance according to a reductionist approach, we should resist the governing view of laws, since it does not seem inevitable to assume such a modal

\footnotetext{
1 As is well known, however, van Fraassen himself strongly opposes the view that one of the main tasks, if not the main, of natural sciences is the search after laws.
} 
feature for the notion of law. In such an framework, it is not a conceptual truth that laws govern, so that it is perfectly possible to entertain a non-governing view of laws without being plainly inconsistent: in this framework "laws are purely descriptive of the particular matters of fact" (Beebee 2000, in Carroll 2004, 256).

For a philosophical position, however, to be logically consistent is not equivalent to be convincing. In the present paper I will argue against this particular descriptive emphasis of the non-governing view of laws, by showing further motivations - in addition to the most familiar ones - why such view of laws turns out to be especially defective. Although it is not plainly inconsistent to deny that the modal character is intrinsic to the notion of law, I will argue that the purely descriptive view has such counterintuitive implications that it fails to countenance features that any decent notion of law cannot, after all, afford to miss. In fact, the only real motivation for defending a descriptive view of laws appears to be a quest for ontological parsimony - the whole reality conceived as nothing but an astronomical amount of unconnected local facts - a motivation that requires a significant price to pay in philosophical terms. On the basis of the claim that laws should be rather viewed as grounding natural processes in a non-purely descriptive fashion, I will then elaborate on one of the possible options that are alternative to the regularity view, namely the so-called primitivist approach to laws: a special attention will be then devoted, in the last section, to the implications that this form of nomic primitivism might have on the issue of the status of the wave function in that particular formulation of quantum mechanics known as Bohmian mechanics.

\section{The descriptive view of laws}

The debate on the nature of lawhood is a complex, intertwined network of issues. Starting from a very general dichotomy, according to which either laws are somehow a part of nature or they pertain only to scientific theories (hence lastly - to us as knowing subjects), the controversy proceeds toward further, 
more specific issues: regularity vs. necessity, the role of Humean supervenience, the relation with the use of laws in scientific practice, the governing vs. the nongoverning status of laws, and so on. Ned Hall claims, for instance:

By far the most central and important question about laws of nature is this: are they mere patterns in the phenomena (patterns that are in some way salient, to be sure - but still, nothing more than patterns)? Or are they something more, something that somehow governs or constrains those phenomena? Disagreement over this issue constitutes the Schism in contemporary philosophical work on laws of nature. (Hall 2012, p. 1$)^{2}$

This sort of disagreement is in non-linear relation with different, possible positions over the issue whether we should think that there really are items like 'laws' and the search for such 'things' is the essence of science: anti-laws philosophers like Bas van Fraassen or Ron Giere have vehemently denied it:

The claims of some philosophers, that scientists seek to discover laws of nature, cannot be taken as a simple description of scientific practice, but must be recognized as part of an interpretation of that practice. The situation is complicated, of course, by the fact that, since the seventeenth century, scientists have themselves used the expression «law of nature» in characterizing their own practice. (Giere 1999, p. 84)

But if one accepts as a working hypothesis the existence of 'law-like' items in the inventory of the world, a major issue is of course what are the terms in which should we conceive the relation between such items and the particular phenomena that in some way or another 'fall under' them. It is at this stage that the controversy 'governing vs. non-governing' arises. The “Governing answer $[\ldots]$ insists that there are genuine laws of nature and furthermore that these laws govern or even produce the events of the world" whereas "the NonGoverning answer $[\ldots]$ has it that there are genuine laws of nature, but that they do not govern or produce the events of the world. The mosaic of events

${ }^{2}$ Hall 2012 is an unpublished manuscript, which is a much more comprehensive version of Hall 2015. 
displays certain patterns, and it is in the features of some of these patterns that we find laws.” (Cohen, Callender 2009, p. 2). In a Humean, reductionist perspective, we should be careful in admitting suspect versions of necessity in nature so that, as a consequence, we should not require from laws any 'governing' role as a constitutive feature. In the global metaphysical view of the world that is on the background of (any version of) views like this, any modal, governing feature in mentioning laws must be traced back to us as subjects, not to the world, that in itself is nothing but a collection of sparse entities, usually conceived as discrete. Hall captures the Lewisian terse way to characterize this view: "Drawing inspiration from Hume, [Lewis] took the fundamental ontological structure to consist in the pattern of instantiation by space-time points of perfectly natural monadic properties, together with the facts about the spatiotemporal relations among those points.” (Hall 2012, p. 5)

In a regularist brand of a reductionist perspective, what is admitted is just the existence of $\mathrm{a}(\mathrm{n}$ astronomical $)$ number of matters of fact and the existence of a certain number of regularities connecting them (Mumford 2004, 32): although it is far from trivial specifying what a regularity exactly is in non-modal terms, a (naïve) regularity view denies the existence of necessary connections or laws whose role in some way or another would be to ground the regularities. The more sophisticated versions of a regularity view, summarized in the so-called Mill-Ramsey-Lewis best-system view, turn out to be an elaboration of how we as subjects should organize regularities, if we want them to play the role that nonHumean views usually associate to the notion of law. The now classic formulation of David Lewis - according to which the 'laws of nature' are the universal statements that belong to all the true deductive systems with a best combination of simplicity and strength (Lewis 1973, 73) - makes explicit the disregard for what is often interpreted as the instrinsically modal character of natural laws. According to Peter Menzies, for instance, this is exactly the main defect of all regularity, best-system approaches to laws:

The defect is that they fail to capture the modal character of laws of nature. [...] It is in virtue of this modal feature of laws that they fail to conform to Humean supervenience: 
two worlds agreeing on actual occurrent facts may differ in their modal structure. Lewis's theory fails because all such regularity must fail; for they try to fashion modal facts from the thin actualistic resources of Humean empiricism. But to do this is to commit what might be called the actualist fallacy of trying to derive conclusions about the possible and the necessary from premises about the actual. No amount of sophisticated talk of ideal systems that combine simplicity and information content will make this fallacious inference virtuous. (Menzies 1993, pp. 200-201)

In the wake of the position echoed by Menzies, there might be then a tempting move for the anti-regularist: by assuming the reliability of a strong modal intuition on laws, the anti-regularist might count a governing view of laws as an instance of this modal intuition and define laws as abstract governing entities, with the effect of dismissing the non-governing view of laws on simple conceptual grounds.

Now, no matter what the position on the irreducibility of modal features of laws we hold, objections of the sort argued for by Menzies look like a petitio principii, since whether laws of nature should be conceived modally or not is exactly the point under discussion. Moreover, a regularist may well resist the claim that the notion of law is to be defined in governing terms: she will try to claim that such move is far from unavoidable and that, as a consequence, a nongoverning conception of laws is perfectly consistent. This is what Helen Beebee, for example, tries to accomplish by claiming that one can define law as governing only by assuming an analogy between laws in nature and laws in other domains such as theology, politics, ethics and so on: but if this assumption is rejected (and it can be rejected), the governing feature of laws fails to be a conceptual truth (Beebee 2000).

According to Beebee the governing intuition about laws might be justified only if we assume a deep similarity between the way in which phenomena are constrained by natural laws on one side and the way in which moral individuals as believers, citizens and so on are subject to prescriptive laws on the other. This similarity fails, however, for at least two reasons. First, in the human, prescriptive domain it is possible for an individual to violate laws, something 
which is not easily the case with natural laws: indeed, it seems constitutive of moral and political laws that they can be broken, since it is from this possibility that their prescriptive role derives its meaning. Second, moral and political laws contemplate forms of punishment for their violation, something which hardly makes sense if extended to natural laws. What does all this show? Well, it is very plausible to think that if common sense does take it to be part of the concept of law of nature that those laws govern, then it does so only because of a tacit assumption that laws of nature operate in a way that is analogous to the way that other laws - laws which really do govern - operate. But that assumption cannot be maintained, since the alleged governing nature of natural laws would have to be entirely unlike the prescriptive nature of moral and other laws. (Beebee 2000, in Carroll 2004, p. 260)

Now even if, as a working hypothesis, we assume the plausibility of these $\operatorname{arguments}^{3}$ and concede that the analogy between natural laws and other sorts of law is not entirely plausible, or implausible at all, the question remains: what exactly does the regularist view implies about the very notion of law? First of all, let us focus on a linguistic stipulation. If by 'law' we agree to mean 'regularity+something-grounding-the-regularity', it follows that in strictly naïve regularist terms there are no laws at all: according to the metaphysical view on the background of the regularist stance, the world is a lawless place. On the other hand, we may simply decide to equate 'law' and 'regularity', without charging the term 'law' with the responsibility of embodying some principles the principles 'in virtue of which' the regularity would be supposed to hold ${ }^{4}$ over and above the regularities themselves. The latter is the option adopted by Beebee 2000 who characterizes the divide between Humean and anti-Humean stances in these terms:

For the anti-Humean, laws (unlike accidentally true generalizations) do something they govern what goes on in the universe - and they therefore require some sort of

\footnotetext{
${ }^{3}$ The issue of possible violations of laws of nature, for instance, is not an idle one.

4. In our opinion the first strategy is definitely less confusing but, provided uniformity is guaranteed, no serious conceptual harm is caused by the second strategy.
} 
ontological basis $[\ldots]$ that gives them this ability. Humeans, on the other hand, do not require laws to "do" anything: like accidentally true generalizations, laws are at bottom merely true descriptions of what goes on. Thus for the Humean there is no need for any ontological distinction between laws and accidents. (Beebee 2000, in Carroll 2004, pp. 258-259)

Under this linguistic stipulation concerning laws, then, let us focus on what in a Humean framework a purely descriptive view of laws might entail. Humeans deny the need for a search after something that might 'ground' a regularity: they "do not require laws to «do» anything: like accidentally true generalizations, laws are at bottom merely true descriptions of what goes on". The descriptive view seems therefore to support the idea that a law is literally nothing but a sum of facts: "for the Humean, since the laws are descriptive, what the laws are depends on what the facts are" (ibidem). What I would like to argue for in what follows is that such view, although it cannot be ruled out in terms of logical consistency, entails features that are seriously contrived in order for the descriptive view of laws to be plausible. I will take into account four points: (1) determinism, (2) the implications of a possible set-theoretic reading of a law-assum, (3) the problem of the cardinality of the set of facts, (4) the problem of uniformity.

\section{(1) The case of determinism}

Let us consider then first the case of deterministic evolution over time. According to Beebee, determinism is useful to express coincisely the nongoverning relation that by a Humean standpoint is supposed to hold between the law and the facts that intuitively are accounted for by the law:

We can characterize determinism in the following rough and ready way: the state of the universe at any given time together with the laws of nature determines what the state of the universe will be at any future time. But what does "determines" mean here? For the Humean, the laws and current facts determine the future facts in a purely logical way: you can deduce facts from current facts plus the laws. And this is just because laws are, in 
part, facts about the future" (Beebee 2000, in Carroll 2004, p. 257, emphasis in the original text).

Two minor points, to begin with, that are far from clear. What would the notion of fact about the future exactly mean by a strictly Humean, descriptivist viewpoint? It seems that, in the metaphysics this viewpoint presupposes, there is simply - at every given instant of time - a given collection of facts, none of which can be 'about' something that does not exist yet. A fact is what it is just because there is nothing beyond it and this is just the role that it is supposed to play in this lawless image of the world: to be nothing but what it is, full stop. Moreover, no matter whether the notion of fact about the future is consistent with a Humean metaphysics, I do not see how a Humean can be legitimated to say that laws are facts but only 'in part': What is the remaining part? Does it need to be 'added' to the purely factual part of laws? Was not the core of the Humean view of laws that these are literally nothing but facts?

But let me come to the main point. This Humean understanding of determinism overlooks that its 'determining' capability is grounded on a specific mathematical formulation of a dynamical law. Take the Newton $2^{\text {nd }}$ law. The general form of this dynamical law states a proportionality between force and acceleration: when a specific formulation for a kind of force is inserted into the $2^{\text {nd }}$ law, we obtain a mathematical equation that, under non-trivial conditions turns out to be integrable. This is what justifies us to assume that the knowledge of a given (initial) state and of what is the force (if any) acting on the system makes it possible to determine future states of the system (and also past states, if the evolution satisfies time reversal invariance). So the crucial point is that the determination is possible due to the functional relations among physical quantities, relations that are encoded into the mathematical formulation of Newton's law: this encoding can hardly be reduced to any 'sum' of facts and cannot be easily accounted for in a purely descriptive view of laws. In addition to that, let us consider the relation between determinism and predictability. There are well-known physical situations (deterministic chaos, three-body problem, ...) in which, apparently, the phenomena are governed by deterministic laws but we 
are not able to predict what the future states will be, namely we are not able to 'produce facts' - in the Humean-like jargon - on the basis of the dynamical law at hand. What is left of the Humean-kind-of-law if we simply cannot deduce the future facts on the basis of the pair <initial conditions, laws even if the phenomena are governed by a deterministic law?

\section{(2) Laws-as sums: a set-theoretical reading?}

The idea that laws are 'sums' of facts lends itself to receive a set-theoretic reading. So let us suppose that we have the following collections of states $\mathrm{S}$ at their respective times $t$

$$
\begin{gathered}
\mathrm{F}_{n}=\left\{\ldots \ldots \ldots, \mathrm{S}\left(t_{n-1}\right), \mathrm{S}\left(t_{n}\right)\right\}, \\
\mathrm{F}_{n+m}=\left\{\ldots \ldots \ldots, \mathrm{S}\left(t_{n}\right), \ldots \ldots, \mathrm{S}\left(t_{n+m-1}\right), \mathrm{S}\left(t_{n+m}\right)\right\}
\end{gathered}
$$

If we assume that any of these states is a collection of values of a set of relevant physical quantities, each state may well represent a 'fact' in the regularist vein, since each state works as a sort of snapshot at its time $t_{*}$ ) of the physical situation at stake. Moreover, we assume that the states both in $\mathrm{F}_{n}$ and $\mathrm{F}_{n+m}$ are obtained as the computational output of the algorithm implicit in one and the same deterministic dynamical law.

Now, the intuition tells us that we have here the same law accounting for the evolution of our system at two different times. But, since in a Humean reading there is nothing to a law except a 'sum' of facts, in the present case the Humean reading seems to have no resource to avoid the paradoxical consequence that here we deal with two sort-of-laws, since we have two different sums! Even worse: at any successive instant of time a new law-as-a-sum is generated. But, then, being parsimonious on any alleged modal features of the world seems paradoxically to imply a wild and uncontrollable generation of laws-as-sums over time, a phenomenon in strong tension with an aspiration to a metaphysical economy. On the other hand, should there be a unifying principle according to which $\mathrm{F}_{n}$ and $\mathrm{F}_{n+m}$ might be shown to be just two instances of one and the same 
law, this principle could not simply supervene on the states and because of this non-supervenience this account would immediately become a non-Humean one.

\section{(3) The problem of cardinality}

An additional problem (but perhaps for another paper) for the regularist view might concern the cardinality of the set of all possible facts. Is that set finite or infinite? Since it seems hard to devise a robust argument to argue that facts are absolutely in finite number, let us turn to the infinity horn. In this case, our intuition is likely to suggest that the set of all possible facts should be conceived as a countable set, but to a large extent what is intuitive or not is a matter of convention: there seems to be no unique way of cutting reality into facts so as to ensure that there are exactly as facts as natural numbers, so who are we to deny that the set of all the facts might have the cardinality of the reals? In this case, however, we might have a hard time simply in discriminating one fact from another, so that even the most basic statement of a regularist view - there are just facts - seems to be under threat.

\section{(4) The problem of uniformity}

Sometimes the above Humean view of a lawless world in terms of 'mosaic' of individual, local matters of fact is formulated with the aid of what has been called recombination (Hall 2015) or redistribution (Hildebrand 2013) principle. Namely, given a mosaic of individual, local matters of fact, there is no privileged way of setting up the mosaic, since this alleged privileged way would amount to a sort of 'ordering' principle over and above the mosaic itself, a consequence that a diehard Humean could not admit: hence any possible combination or distribution of such matters of fact is equally possible. In this case, however, a ban on any constraints over the possible arrangements of individual, local matters of fact seems to have the undesirable consequence that it fails to explain why our world is in fact highly uniform, since the absence of constraints makes non-uniform arrangements as likely as the uniform ones. The recourse to the idea of pattern is of no help here: if we have sufficient reasons to ascribe one or more 'patterns' to a 
given arrangement, those patterns embody de facto a kind of uniformity that the no-constraints ban is unable to account for.

\section{Primitivism about laws}

As we have seen, there are a number of reasons why one should be dissatisfied with the anti-necessitarian viewpoint. In particular, from that viewpoint it turns out to be difficult to cope with that seemingly irreducibly modal aspect that informs our explicit and implicit way of employing or referring to laws: for instance, the demand according to which laws must cover 'possibilities', and not just actualities, and the demand according to which, when we ask a law to explain facts, we search after what is in virtue of which facts obtain. Moreover, a look at the actual scientific practice when analyzing how laws work does nothing but increase the dissatisfaction: one of the most perplexing points of the above discussed features of a Humean stance on laws of nature is that of implying a truly structureless world, an implication that seems hard to reconcile with a scientific image of the natural world, even broadly construed.

It is from this perspective that we will be concerned with an alternative to the descriptive view of laws, namely the so-called primitivist approach ${ }^{5}$. Basically, primitivism about laws can come in two varieties: in its metaphysical dimension, laws belong to the fundamental inventory of the world whereas in its conceptual dimension, laws are not to be reduced to more primitive notions:

My analysis of laws is no analysis at all. Rather, I suggest we accept laws as fundamental entities in our ontology. Or, speaking at the conceptual level, the notion of a law cannot be reduced to other more primitive notions" (Maudlin 2007, p. 18).

\footnotetext{
${ }^{5}$ Under the assumption according to which a descriptive view of laws is a reductionist view in a serious sense, the primitivist approach is an anti-reductionist view which includes similar, but mutually non-equivalent positions such as Carroll (1994, 2008), Lange (2000, 2009), and Maudlin (2007): in the present paper we will focus on the Maudlin version.
} 
When a given notion $N_{\mathrm{i}}$ is chosen as primitive within a set $\left\{N_{1}, \ldots, N_{\mathrm{i}}, \ldots, N_{\mathrm{n}}\right\}$ of possible alternatives, the general question arises immediately of what would be the deeper motivation for ascribing to $N_{\mathrm{i}}$ rather than to $N_{\mathrm{i}+1}$ or $N_{\mathrm{i}-1}$ the status of 'primitive', a question the Maudlin's reply to which reads as follows:

Taking laws as primitives may appear to be simple surrender in the face of a philosophical puzzle. But every account must have primitives. The account must be judged on the clarity of the inferences that the primitives warrant and on the degree of systematization they reveal among our pre-analytic inferences. (Maudlin 2007, p. 15) ${ }^{6}$

On the background of the obvious remark, then, according to which there must be some entity or notion that is selected as primitive anyway, the choice of laws as primitive may have at least two significant strong points. First, the primitive status of laws allows one to have a more effective and stimulating confrontation with the role of laws within specific scientific theories: the above reference to actual scientific practice should be read in this sense. Second, the primitive status of laws promises to plausibly accommodate interrelated notions - causation, explanation, counterfactuals - that, together with lawhood, appear to form a true conceptual network. In fact, laws as primitives can be reasonably seen as able to translate causal relations into nomic ones, as grounding counterfactuals if similarity of possible worlds is formulated in terms of compatibility with given laws and, finally, they can preserve their role in explanation. Let us briefly focus on the two last points.

The connection between laws and counterfactuals is obviously deep, due to the issue of whether modality is intrinsic to the very notion of law or not. As we recalled earlier, laws are intuitively supposed to account not only for the actual phenomena but also for possible ones, and are supposed to account for the uniformity of the natural world in terms of non-contingent, law-governed processes. In the Maudlin version of primitivism, laws as primitives can handle

\footnotetext{
${ }^{6}$ In his proposal, Maudlin selects a specific form of fundamental law as particularly apt to cover many law instances in the special sciences, namely what he calls FLOTE, i.e. Fundamental Law Of Temporal Evolution (Maudlin 2007, p. 12). This option raises immediately the question (that we will not touch here) of whether this choice compels one to assume also time - entering the FLOTE-kind of laws - as a primitive entity or not.
} 
this modal core of the lawhood intuition through their capacity of generating (classes of) models. A given law gives rise to possible worlds to the extent that it sets boundaries to the occurrence of phenomena. All that according to precise prescriptions fixed by the law remains within the boundaries is 'possible': "the possible worlds consistent with a set of laws are described by the models of a theory that formulates those laws" (Maudlin 2007, p. 18). If setting the boundaries for the validity of a law is what allows for possibility in the primitivist framework, necessity is obtained at a very low cost: since it is the very compatibility with laws that generates a set of models, the laws themselves must hold in all models of the set and therefore display a nomic necessity (in the usual, possible-world language, Maudlin 2007, p. 21)7. As to the connection with explanation, the primitive status of laws allows us to select any account of explanation we like in which laws play a sufficiently crucial role, without worrying whether the plausibility of the model of explanation we selected is threatened or not by some more fundamental notion in terms of which the notion of law is reduced: we have explanation of an event $f$ whenever we have a nomic subsumption of $f$ under the relevant set of laws $L$, namely whenever we may show that, given $L$, the event $f$ is what we should expect (Maudlin 2007, pp. 34 ff).

Since no wide-ranging philosophical view concerning such a deep issue as the issue of laws on nature can go unchallenged, let us take into consideration some possible objections to the primitivist account. A first point concerns the status of laws in terms of their alleged 'fundamentality'. At a given stage of development of a scientific theory, we may have reasons to think that a given law is fundamental, a circumstance that seems to go along well with the claim that laws are primitive endowments of the natural world's ontology. History of science, however, has taught us that laws that were supposed to be fundamental turned out to be only special cases of more general laws and still history of science,

\footnotetext{
7 An additional advantage seems to be that, if we assume laws as primitives, the principle according to which we generate models only in terms of their compatibility with laws requires so to say - a 'minimum' of modality: possibility is exactly law-compatibility, whereas necessity is obtained simply from the fact that the validity of the law is a sort of fixed point in any lawgenerated model.
} 
jointly with philosophy of science, suggests that there are no reasons to think that there is a foreseeable end to this process of ever-increasing generality. How are we to cope with this problem? It hardly looks reasonable to assume that the whole network of laws is primitive from the start: would not it be awkward to suppose that, for instance, Kepler's laws are as primitive as Newton's laws, and these in turn as primitive as the Einstein's field equations of general relativity? Although clearly primitive and fundamental are not equivalent concepts, one might think that the conventionality inherent in selecting which laws are supposed to be fundamental and which derivative might be at least disturbing for primitivism. On the other hand, in different areas of science results have been obtained that prove in principle our inability to grasp some kind of knowledge from the undecidability theorems to the black hole information loss theorems so that the idea that we might be unable to access part or the totality of the really fundamental laws need not contradict the possibly primitive ontological status of some of these laws.

A second point has been raised concerning the alleged failure of the primitivist account in its explanatory power. According to Tyler Hildebrand, for instance, the primitivist approach fails in this respect exactly like Humeanism (Hildebrand 2013). As we recalled earlier Humeanism - in accepting any distribution of properties as possible (according to the recombination principle) has no resources to explain the circumstance that there seems to be a certain uniformity among distributions and that not all distributions seem to be on a par in this respect. Hildebrand argues that primitivism about laws suffers from an essentially identical problem: according to Hildebrand, taking laws as primitives has the two following, significant implications:

First, PGL [what Hildebrand calls the "Primitive Governing Laws" view] allows no explanation of why we have the laws we do as opposed to other possible laws. If laws are wholly primitive, on what basis can we explain why we have one rather that another? [...] Second, PGL places no restrictions on the content of laws, so laws could give rise to every logically consistent distribution of natural properties. (Hildebrand 2013, p. 5) 
A preliminary, meta-philosophical kind of remark is that a principled theory concerning natural laws is supposed to address a de iure problem - namely, a problem like "what is a law of nature?" or "how does a Humean or a non-Humean view contribute to a deeper understanding of laws of nature?" - and not a de facto problem, like the contingent problem of why we have laws that set constraints for certain systems, quantities or processes and not for others. For this reason, the latter kind of problem is something for which primitivism need not provide an independent explanation.

In a more substantial vein, Humeanism and primitivism are far from having the same problem. While Humeanism - due to the anti-necessitarian flavor of the adopted recombination principle - is forced not to have resources to privilege certain distributions over others, primitivism is not: in assuming laws as belonging to the metaphysical, fundamental inventory of the world, I hold that primitivism has the freedom to choose the particular way in which laws are supposed to be primitive. In this sense, I propose to include in the irreducible property of being primitive for a law the very fact that certain distributions of natural properties are privileged over others: otherwise, what does a natural law stand for, if not for dictating a certain nomological structure for (a portion of) the natural world? According to this proposal, taking laws to be primitive in the above sense might recall the Strawsonian sort of solution to the induction problem, according to which there cannot be any question as to the rationality of the employment of induction, since by being rational we mean, amongst other things, using induction. The primitive status of laws would be exactly what gives us the right to consider laws as governing entities, since imposing a structure over the whole set of possible distributions of natural properties is one of the features by which we conceive nomicity for the natural world ${ }^{8}$. In other words, nothing in the formulation of PGL prevents that, if we allow PGL itself its Strawsonian role, we might see it somehow negotiating with natural reality so as to incline toward certain arrangement of natural properties instead of others.

${ }^{8}$ I said one of the features and not the feature, otherwise I would have turned back to the above mentioned claim according to which we should define laws in that way, a claim that - as I have recalled earlier - Humeans can consistently resist. 


\section{Nomic primitivism and the interpretation of the wave function in Bohmian mechanics}

In addition to its interest by a strictly philosophical viewpoint, the primitivism on laws of nature may have a significant connection with a recent option discussed in the area of the foundations of contemporary physics, and in particular within the issue of the status of the wave function (usually denoted by the Greek letter $\psi$ ) in quantum theory, in fact, one of the most controversial issues in that area (Albert, Ney 2013). As far as the usual meaning attached to the traditional $\psi$ in standard quantum mechanics is concerned, a 'wave function' $\psi_{S}$ is, according to textbook presentations, a formal tool that can be assigned to a single microsystem $S$ and whose main role is to enable an observer to calculate the probability of obtaining one of the permissible outcomes in a well-defined experimental setting. There was a time in which some of the founding fathers of quantum theory - from Schrödinger to De Broglie, from the early Born to Einstein - entertained the idea that the wave function could be some sort of physical entity in its own right and not just a convenient computational tool. As a matter of fact, it soon became clear that the peculiar status of the mathematical space in which the wave function lives prevented this idea to hold in naïvely realistic terms and the wave function acquired rapidly the purely informational role that the orthodox formulation of quantum mechanics attributed to it for decades. In more recent years, however, the obsolescence of the Copenhagen orthodoxy and the flowering of interpretational analysis of quantum mechanics led to a growing dissatisfaction with a merely epistemic view of the wave function, so as to push the pendulum toward radical views in the opposite direction: the most notable example is the wave function ontology or configuration space realism (Albert 1996), according to which "the fundamental space in which entities evolve is not three-dimensional, but instead $3 \mathrm{~N}$-dimensional, where $N$ is the total number of particles standardly thought to exist in the threedimensional universe" (Monton 2002, p. 265). 
In a particular formulation of quantum mechanics, namely Bohmian mechanics, an interpretation has been proposed according to which the wave function can be conceived as a nomological entity, namely an entity "more in the nature of a law than a concrete physical reality" (Goldstein, Zanghì 2013, p. 96). This interpretation proposes somehow a shift from the question of the nature of the wave function to the question of its role: namely the issue is not what kind of object the wave function is but rather what the wave function does for grounding the ontology in a nomological sense (Dürr, Goldstein, Zanghì 2013, Goldstein, Zanghì 2013)9 ${ }^{9}$. It is this nomological interpretation that naturally raises the question of how and to what extent this foundational debate concerning quantum theory may connect with the above considerations on the status of natural laws In particular, two basic questions arise:

1) How exactly should we understand the meaning of 'nomological'?

2) Under the hypothesis that such meaning can be made sufficiently clear, can such an entity as a 'nomological' one be really thought of as part of the physical world and in what sense?

Standard Bohmian mechanics is an observer-free formulation of (nonrelativistic) quantum mechanics, according to which the latter describes quantum particles and their trajectories in physical space and time: in doing this, Bohmian mechanics is said to provide a space-time ontology of non-relativistic quantum mechanics, namely a class of well-specified kind of objects and properties displayed in space-time that quantum mechanics is supposed to be about: being the primary target of the theory, this space-time ontology is called primitive ontology ${ }^{10}$. In this framework, the wave function plays a crucial role: in addition to satisfying the Schrödinger's equation, the wave function determines the particles' motion via the especially Bohmian addition to the ordinary structure of

\footnotetext{
${ }^{9}$ It is in this sense that Bohmian mechanics might be interpreted as "en-theorizing" a form of nomological realism for the wave function, similarly to what several years ago Arthur Fine proposed in order to assess the very nature of the Einsteinian use of such notions as 'realism', 'causality' and 'determinism' (Fine 1986). For some remarks on a more general en-theorizing strategy concerning BM, see Laudisa 2012.

10 There is now an extended literature on the details of such an ontology and its connections with many of the general, interpretive issues in the foundations of non-relativistic quantum mechanics, and this is not the place to provide a review. For a recent presentation and re-assessment, see Dürr, Goldstein, Zanghì 2013.
} 
quantum mechanics, namely the so-called guiding equation. On the basis both of this central role of the wave function in its basic formulation and its generically 'realistic' flavor - the theory is 'observer-free' to the extent to which all observation-related notions are derivative and not primary in the theory - a decisive question that deals with the very foundations of Bohmian mechanics is then how to make sense of the wave function, without incurring either into the Schylla of a purely instrumental view of it on one side or into the Charybdis of a heavy 'object-like' view of it on the other.

As far as the usual meaning attached to the traditional $\psi$ in standard quantum mechanics is concerned, a 'wave function' $\psi_{S}$ is, according to textbook presentations, a formal tool that can be assigned to a single microsystem $S$ and whose main role is to enable an observer to calculate the probability of obtaining one of the permissible outcomes in a well-defined experimental setting. If we start thinking differently about the $\psi_{S}$, however, as is the case with the Everett interpretation or the Bohmian mechanics, the only system $S$ to which a $\psi_{S}$ can be genuinely attached is the whole universe, a circumstance that yields deep implications. In Bohmian mechanics the wave function of the universe - let us denote it with $\Psi$ - plays a crucial role at different levels. It is $\Psi$ that the basic equations of the theory govern in a fundamental sense and when we discuss the status of the wave function as a nomological entity, it is $\Psi$ that we are primarily talking about.

Within this framework, conceiving the $\Psi$ in nomological terms presents some remarkable advantages. First, the nomological interpretation allows one to justify the circumstance according to which the particles' motion is affected by the wave function without affecting it back: when we think of a 'law' determining in some sense the phenomena falling under its application domain, we do not expect any reaction behavior by the law-governed phenomena on the law itself! Second, the nomological interpretation seems able to accommodate more naturally the celebrated problem of time in quantum cosmology. If the $\Psi$ as a law is supposed to aptly govern the particles' motion and the related properties, only the latter are supposed to change over time and not the wave function: if it is a 
law that governs the way in which things in the world change, we expect the law-governed things to depend on time, not the law itself: "from a Bohmian perspective, the timelessness of $\Psi$ is not a problem. Rather, it is just what the doctor ordered." (Goldstein, Zanghì 2013, p. 100). Third, the nomological interpretation seems especially suitable for a further feature of Bohmian mechanics, namely the possibility to recover from $\Psi$ the wave functions $\psi_{S^{\prime}}$, $\psi_{S^{\prime \prime}}, \ldots$, that can be assigned to well-defined subsystems $S^{\prime}, S^{\prime \prime}, \ldots$ of the universe in a suitable sense. This possibility obtains in Bohmian mechanics through the definition of the so-called conditional wave function: I argue that this definition, in addition to allowing a well-defined notion of a subsystem of the universe, is a further instance of what it might mean for the $\Psi$ to be mainly a nomological entity. Let $\Psi_{t}$ be the wave function of the universe at time $t$ and let $S$ be a specific system under scrutiny (clearly, $S$ is also a subsystem of the universe whose wave function is $\Psi$ ). Let us suppose further to decompose the configuration of the universe $Q=(X, Y)$ into the configuration $X$ of the system $S$ and the configuration $Y$ of the rest of the universe, something that we may call the 'environment' of $S$. Then we define the conditional wave function of $\mathrm{S}$ at time $t$ by the expression

$$
\psi_{t}(x)=\Psi_{t}(x, \Upsilon)
$$

A remarkable implication of this definition is that, under suitable conditions, the conditional wave function of $S$ does indeed satisfy the Schrödinger's equation for $S$, namely, given the wave function of the universe, each of the wave functions $\psi_{S^{\prime}}, \psi_{S^{\prime \prime}}, \ldots$ of the subsystems $S^{\prime}, S^{\prime \prime}, \ldots$ of the universe behaves just like ordinary quantum mechanics prescribes they should phenomenologically behave ${ }^{11}$.

But in addition to the 'conservation' of the state phenomenology, which is an important property anyway, there is a further point that is directly relevant to our present philosophical discussion: the joint action of the $\Psi$ and the primitive ontology in fixing the state of the ordinary systems that we deal with. Suppose an observer $O$ who is operating with a specific system $S$, on which she is about to

11 A rigorous justification that the conditional wave function is phenomenologically 'wellbehaved' can be found in Dürr, Goldstein, Zanghì 1992. 
perform a measurement. By assuming a primitive ontology of actual configurations that are independent from preparation and measurement procedures, BM allows $O$ to concentrate on $S$ by putting to work the whole galaxy of the space-time locations of all the subsystems that are ontologically fixed anyway but that are not directly relevant to the measurement on $S:$ in a sense, the whole ontological web of the (per se existing) space-time locations works as a fixed parameter, except for the $x$ that figures as argument of the conditional wave function $\psi(x)$. But if we agree that the specification of states for subsystems of the universe is a fundamental nomological task of a theory with a wide scope such as quantum mechanics is usually assumed to have, the nomological role that the wave function takes on in BM is apparent here. The $\Psi$ does not belong to the basic endowment of primitive ontology, but it is in its 'interplay' with primitive ontology that it expresses its role of state determination: no direct entity-realism concerning the wave function is assumed, but rather a primitive-ontology-based theory in which a precise story is told on how the wave function - although not belonging itself sic et simpliciter to the inventory of primitive ontology - implements an observer-free description of quantum systems in terms of such primitive ontology.

On the background of our preceding discussion on nomic primitivism, at least two general points are in order. First, the general view of laws as primitive entities may turn out to be well-suited to the role that the wave function as-alaw occupies in Bohmian mechanics, since such role appears not reducible to anything more fundamental in the overall structure of the theory. Second, the specific nomic role that the wave function plays in Bohmian mechanics may exemplify the idea that a nomic entity like a wave function in Bohmian mechanics can be 'part of an ontology without being necessarily conceived as an ordinary, stuff-like object. As we have seen, the wave function is not in itself a piece of primitive ontology, but it plays the role of generating and making possible the 'displacement' of primitive ontology and its features in space-time. The picture that emerges is, so to say, two-dimensional: the entities included in the primitive ontology belong to the dimension of what fundamentally exists in space-time, but the wave function belongs to the dimension of what tells 
primitive ontology how to behave in space-time. Therefore, a sensible way to do justice in philosophical terms to this role of the wave function is to take the wave function itself as primitive, although in a nomic sense.

But what is this 'two-dimensional' talk I am referring to? Basically, it is a way to contemplate two different fashions in which something can be part of physical reality according to a Bohmian perspective: the first pertaining to the primitive ontology, the second to the wave function as-a-law. Unless we allow ourselves to distinguish at least these two different ways of being part of physical reality, we can hardly avoid the conclusion that the wave function, if it is to be something more than a computational tool, is real to the same extent as the entities of the primitive ontology, something that in Bohmian mechanics makes no sense. It might be correct to claim, as Esfeld et al. 2014 do, that if we ground the nomological interpretation of the wave function onto nomic primitivism "there is no sharp distinction between a nomological and an ontological interpretation of the wave function, since laws belong to the stock of physical reality as well" (Esfeld et al. 2014, p. 780). But no sharp distinction does not imply no distinction at all. We may think to have a sort of plane, spanned by two dimensions: that of the primitive ontology entities $\mathrm{X}_{\mathrm{PO}}$ and that of the nomological entities $\mathrm{Y}_{\mathrm{N}}$ : each point in the $\mathrm{X}^{\mathrm{PO}}-\mathrm{Y}^{\mathrm{N}}$ plane represents an implementation of a possible behavior of PO in terms of a nomological entity (like the wave function). It is plausible to hold that what the theory is about is not just PO but PO plus the nomological entities in terms of which PO behaves, and nomological entities such as the wave function are essential in order for the behavior of PO to be described by the theory: as Esfeld et al. themselves say "we should be open-minded enough to concede that the ontology of a physical theory may contain other kinds of objects than just particles and fields" (Esfeld et al. 2014, p. 778)12.

\footnotetext{
${ }^{12}$ In their recent paper, Esfeld, Lazarovici, Hubert and Dürr discuss two different options about the lawhood intuition that is supposed to ground the nomological interpretation of the wave function in Bohmian mechanics: "the Humean one that regards the law as a contingent regularity and the one that anchors the law in a disposition of motion of the particles" (Esfeld et al. 2013). In my opinion, neither is convincing and more persuasive than the primitivist option, but a detailed analysis and support for this claim are outside the scope of the present paper.
} 


\section{Conclusions}

On the $6^{\text {th }}$ of April, 1922 Albert Einstein was invited to join at the Société Française de Philosophie. Asked of his opinion about the relation of his relativity theory with the epistemology of Ernst Mach - an epistemology which is remarkably similar to the regularist framework in important respects - Einstein replied:

There does not appear to be a great relation from the logical point of view between the theory of relativity and Mach's theory. For Mach, there are two points to distinguish: on one hand there are the immediate data of experience, things we cannot touch; on the other there are concepts which we can modify. Mach's system studies the existing relations between data of experience; for Mach, science is the totality of these relations. That point of view is wrong and, in fact, what Mach has done is to make a catalogue, not a system. (Einstein 1923, p. 253, emphasis added)

What in memory of the Einsteinian claim we can also call the catalogue view of laws proposed by the regularists turns out to be implausible for analogous reasons. This view is logically consistent but implausible in that it misses what makes a scientific theory of natural phenomena a system rather than a list. It is not true that to assume that there is an ordered structure for phenomena - that order being a consequence of the operations of laws - is a requirement defensible only in analogy with the use of the notion of law in other domains: it is simply that nature turns out to be incomprehensible without such a structure. 


\section{References}

Albert D. 1996, "Elementary Quantum Metaphysics", in J. Cushing, A. Fine, S. Goldstein (eds.), Bohmian Mechanics and Quantum Theory: An Appraisal, Dordrecht, Kluwer, pp. 277-284.

Albert D., Ney A. (eds.) 2013, The Wave Function. Essays on the Metaphysics of Quantum Mechanics, Oxford University Press, Oxford.

Beebee H. 2000, "The Non-Governing Conception of Laws of Nature", Philosophy and Phenomenological Research 61 (3), pp. 571-594 (reprinted in J. Carroll (ed.), Readings on Laws of Nature, University of Pittsburgh Press, Pittsburgh 2004, pp. 250-276, page references are to the reprinted edition).

Carroll J. 1994, Laws of Nature, Cambridge University Press, Cambridge.

Carroll J. 2008, "Nailed to Hume's Cross?”, in J. Hawthorne, T. Sider and D. Zimmerman, (eds.), Contemporary Debates in Metaphysics, Basil Blackwell, Oxford.

Cohen J., Callender C. 2009, "A Better Best System Account of Lawhood", Philosophical Studies, 145, pp. 1-34.

Dürr D., Goldstein S., Zanghì N. 1992, "Quantum Equilibrium and the Origin of Absolute Uncertainty", Journal of Statistical Physics 67, pp. 843-907.

Dürr D., Goldstein S., Zanghì N. 2013, Quantum Physics without Quantum Philosophy, Berlin, Springer.

Esfeld M., Lazarovici D., Hubert M., Dürr D. 2014, “The Ontology of Bohmian Mechanics”, British Journal for the Philosophy of Science 65, pp. 773-796.

Fine A. 1996, The Shaky Game: Einstein, Realism and the Quantum Theory, Chicago, University of Chicago Press.

Frank P. 1949, "Einstein’s Philosophy of Science”, Review of Modern Physics 21, pp. 349-355.

Giere R. 1999, Science Without Laws, University of Chicago Press, Chicago.

Hall N. 2012, "Humean Reductionism About Laws of Nature", Unpublished manuscript.

Goldstein S., Zanghì N. 2013, "Reality and the Role of the Wave Function in Quantum Theory”, in Albert, Ney 2013, pp. 91-109. 
Hall N. 2015, "Humean Reductionism About Laws of Nature", in B. Loewer, J. Schaffer (eds.), A Companion to David Lewis, Wiley \& Sons, pp. 262-277.

Hildebrand T. 2013, “Can Primitive Laws Explain?”, Philosophers' Imprint, 13, pp. $1-15$.

Lange M. 2000, Natural Laws in Scientific Practice, Oxford University Press, Oxford.

Lange M., 2009, Laws and Lawmakers, Oxford University Press, New York.

Laudisa F. 2012, “The Uninvited Guest: 'Local Realism' and the Bell Theorem”, in De Regt H., Hartmann S., Okasha S. (eds.), EPSA Philosophy of Science: Amsterdam 2009, Springer, Berlin, pp. 137-150.

Lewis D.K. 1973, Counterfactuals, Harvard University Press, Cambridge Mass.

Maudlin T. 2007, Metaphysics within Physics, Oxford University Press, New York.

Menzies P. 1993, "Laws, Modality, and Humean Supervenience”, in J. Bacon, K.

Campbell \& L. Reinhardt (eds.), Ontology, Causality and Mind: Essays in Honour of

D. M. Armstrong, Cambridge University Press, Cambridge, pp. 195-224.

Monton B. 2002, "Wave Function Ontology”, Synthese 130, pp. 256-277.

Mumford S. 2004, Laws in Nature, Routledge, London.

Van Fraassen B. 1989, Laws and Symmetry, Clarendon Press, Oxford. 